\title{
Análisis de Metodologías de Estudio en Química Inorgánica
}

\author{
M.G. de Bullaude ${ }^{1}$, L.M. Cordoba ${ }^{2}$, M.C. Torres ${ }^{2}$ y J.A. de Morán ${ }^{2}$ \\ Universidad Nacional de Tucumán, (1) Facultad de Agronomía y Zootecnia, (2) Facultad de \\ Bioquímica, Química y Farmacia, Ayacucho N491, (4000) S.M. de Tucumán - Argentina
}

\begin{abstract}
Resumen
El objetivo de este trabajo fue analizar los hábitos y estrategias de estudio de estudiantes de Química Inorgánica en una Universidad argentina. Para evaluar las habilidades operativas de estudio, se aplicó encuestas y cuestionarios a dos grupos de estudiantes. El análisis de estos instrumentos de evaluación permite establecer que los estudiantes de Química Inorgánica no aplican metodologías adecuadas de trabajo intelectual en el estudio. Una de las razones para esta situación parece ser las inadecuadas metodologías de enseñanza-aprendizaje a las que los estudiantes han sido expuestos durante sus previos años de estudio, y que no favorecen el desarrollo de sus habilidades metacognitivas. Los resultados de este trabajo hacen también evidente que se hace necesario incorporar metodologías educacionales que favorezcan la adquisición de hábitos eficientes de estudio.
\end{abstract}

Palabras clave: enseñanza-aprendizaje, aprendizaje de química, metodologías de estudio, estrategias de estudio

\section{Methodologies of Study of Inorganic Chemistry}

\begin{abstract}
The objective of this work is to analyze habits and strategies of study of university students in Inorganic Chemistry courses of an argentinean University. To evaluate the operative study abilities, surveys and tests were applied to two groups of students. The analysis of these evaluation instruments allow to establish that students of Inorganic Chemistry do not apply adequate methodologies in their studies. One of the reasons for this situation seems to be the inadequate teaching-learning techniques to which the students have been exposed during their previous years of study, which do not favor the development of their metacognitive abilities. The results also make evident that educational methodologies in favor on the acquisition of efficient habits of study need to be implemented.
\end{abstract}

Keywords: teaching-learning, chemistry learning, study methodologies, strategies of study

Nota: este artículo está tomado de "Información Tecnológica" [ISSN 0716-8756], vol. 11(3), 129-133 (2000) 


\section{INTRODUCCION}

La evolución de la Química hacia aspectos estructurales, de enlace y termodinámicos durante las primeras décadas de este siglo, indujo a un nuevo enfoque de la enseñanza. Se enfatizó excesivamente, la teoría pura, relegando la Química descriptiva a un segundo plano. Sin embargo, en el presente se reconoce nuevamente la relevancia de los aspectos descriptivos de la disciplina.

Un campo que ha tenido en los últimos años una enorme expansión es la Química Inorgánica. No sólo ha avanzado vertiginosamente la preparación de nuevos compuestos y el estudio de sus propiedades, sino que han surgido nuevas áreas como la ciencia de los materiales, la química bioinorgánica, etc. (Butler y Harrod, 1992). La cantidad de información disponible sobre Química Inorgánica aumenta en forma constante, tanto en los aspectos teóricos como en los experimentales y tecnológicos. Para los estudiantes universitarios puede resultar, por ello, difícil alcanzar un buen nivel de dominio de Química Inorgánica. Es muy importante, en consecuencia, que el alumno que intenta aprender Química Inorgánica aplique algún tipo de metodología de estudio. La misma supone un conjunto más o menos sistemático de pautas que optimizan el aprendizaje, y que se traducen en estrategias concretas. En el presente trabajo se discute la aplicación de técnicas de estudios y estrategias de aprendizaje para el estudio de la Química Inorgánica, y se analizan los resultados de las mismas.

\section{METODOLOGÍA}

Se trabajó con dos grupos de alumnos de Química Inorgánica;

A) Alumnos "regulares", que habían obtenido las calificaciones requeridas para aprobar los trabajos prácticos de la asignatura. La muestra analizada fue de 26 alumnos, y la población total de 150.

B) Alumnos "libres" que por nos haber alcanzado calificaciones aceptables habían dejado de ser alumnos regulares y debían cursar nuevamente la asignatura. La muestra fue de 36 alumnos.

El procedimiento incluyó las siguientes etapas:

Aplicación de un test: Se aplicó a ambos grupos, por separado, al finalizar el dictado de la asignatura, el test de Pózar para evaluar habilidades operativas para el estudio.

Evaluación: A través de la evaluación de los resultados del test de Pózar se obtuvieron datos sobre algunos aspectos de los hábitos de estudio de los alumnos: a) ambientación; b) planificación; c) elaboración; d) asimilación

Encuestas: 1) Se aplicó en el grupo B una encuesta para conocer las opiniones de los estudiantes respecto a las posibles causas de su poco rendimiento, y a los factores que consideraban importante para mejorarlo, 2) Se efectuaron entrevistas con alumnos que habían rendido el examen final, algunos de ellos aprobados, y otros reprobados.

Información: Sobre la base de los resultados obtenidos, se informó a grupos reducidos de alumnos respecto a sus falencias, sugiriéndoles técnicas para sistematizar su trabajo intelectual

Taller: Se implementó para los alumnos "libres" un taller cuyo objetivo fue ejercitar a los estudiantes en metodologías de estudio.

\section{RESULTADOS Y DISCUSIÓN}

A continuación se muestran los resultados obtenidos luego de aplicar la metodología anteriormente descrita. 
En la figura 1 se grafica para cada grupo la frecuencia de los resultados obtenidos en el test de Pózar, expresados en una escala de 1 a 10.

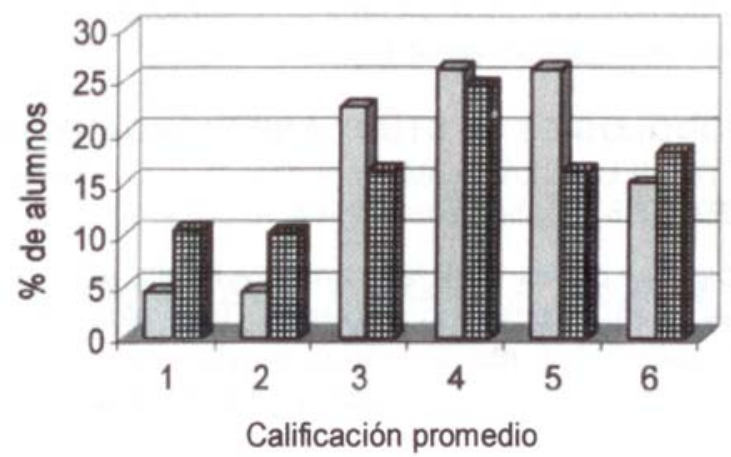

Fig. 1 : Calificaciones promedio en el Test de Pózar (-Grupo A; $\square$ Grupo B)

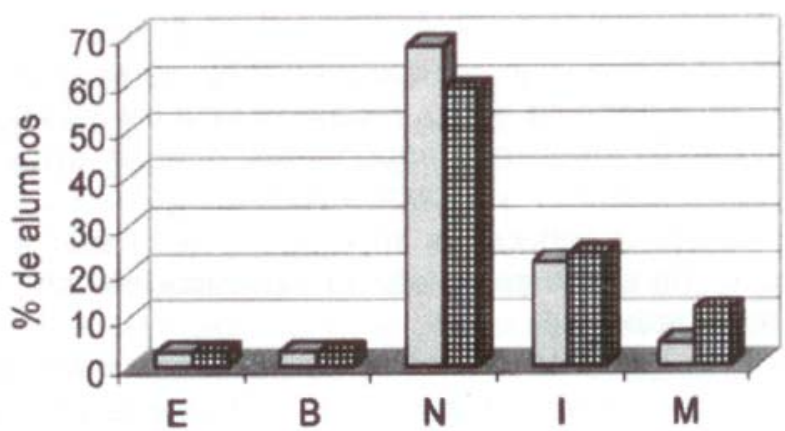

Fig. 2: Nivel alcanzado en habilidades para el estudio (-Grupo A; $\square$ Grupo B)

Se observa que:

a) Las calificaciones promedio son bajas para los dos grupos. La máxima calificación obtenida es 6.

b) Las calificaciones de mayor frecuencia son en general más altas para el grupo A que para el B.

En la figura 2 se representa el nivel global alcanzado en el Test de Pózar, calificado como excelente $(E)$, bueno $(B)$, normal $(N)$, insuficiente $(I)$, malo $(M)$. Se observa que ningún alumno posee hábitos de estudio que puedan calificarse como buenos o excelentes.

Calificación promedio en el test de Pózar para cada serie de preguntas:

La figura 3 muestra las calificaciones promedio obtenidas para cada serie de preguntas, las que corresponden a los distintos aspectos de la metodología de estudio; representando en dicha figura:

- Serie I: Ambientación

- Serie II: Planificación

- Serie III: Elaboración

- Serie IV: Asimilación

- Serie V: Sinceridad

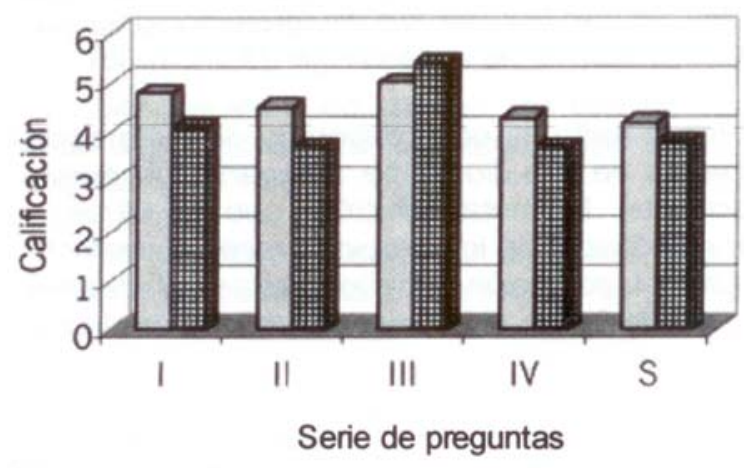

Fig. 3: Calificaciones promedio para cada serie de preguntas

(-Grupo A; $\square$ Grupo B)

Los resultados son mejores para los estudiantes del grupo A que para los de B con respecto a ambientación, planificación y asimilación: y más bajos en cuanto a elaboración.

Respecto a las preguntas de la serie $\mathrm{S}$, (escala adicional) mediante las que se intenta evaluar la sinceridad de las respuestas, los resultados son mejores para el grupo A. 


\section{Resultados de la Encuesta}

Causas del baja rendimiento: En la figura 4 se representan los resultados obtenidos en la pregunta referente a las posibles causas del bajo rendimiento. Las respuestas son: no sabe (NS), falta de estudio (FE), problemas personales (PP), falta de memoria (FM), falta de comprensión (FC). El mayor porcentaje de alumnos atribuye el bajo rendimiento a la falta de estudio. Otra causa señalada como importante es la falta de memoria. Son muy pocos los que mencionan la falta de comprensión.

Factores para un buen rendimiento: Los resultados representados en la figura 5 corresponden a los siguientes factores que los alumnos mencionan como determinantes de un buen rendimiento: saber estudiar (SE), dedicación (Dd); empleo de textos adecuados (TA), buenas clases (BC), buen profesor (BP).El factor que los alumnos consideran más importante es "saber estudiar". Mencionan también como causa de un buen rendimiento la dedicación al estudio.

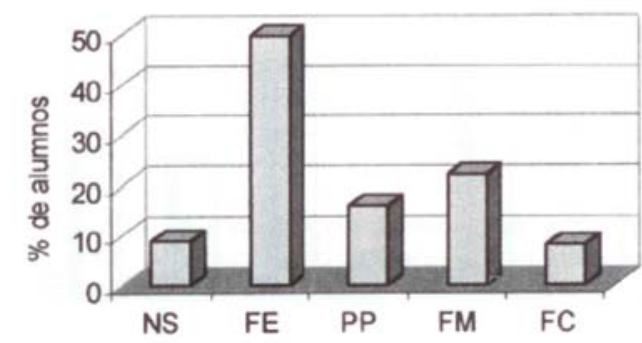

Fig. 4: Opiniones de los alumnos sobre las causas del bajo rendimiento

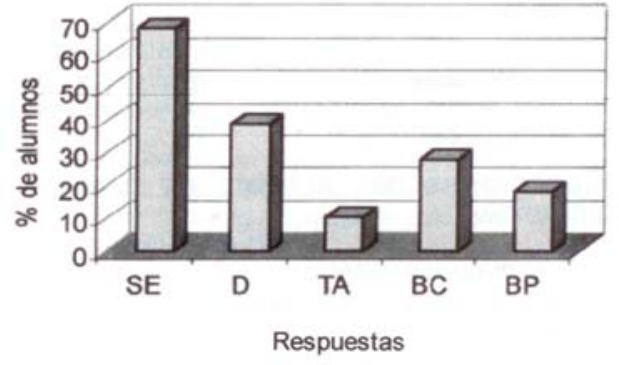

Fig. 5: Opiniones de los alumnos sobre los factores para el buen rendimiento

Etapas necesarias para el estudio: Los resultados representados en la figura 6 corresponden a respuestas de los alumnos sobre las etapas necesarias para el estudio de la química: asistencia a clase $(A C I)$, Lectura rápida (LR), lectura analítica $(L A)$, resumen ${ }^{\circledR}$ y repaso $(R e)$. Para la mayoría de los estudiantes la etapa más importante es la clase magistral, y en segundo lugar la lectura analítica. Un tercio de los alumnos considera importante hacer un resumen y un repaso; muy pocos mencionan la lectura rápida inicial.

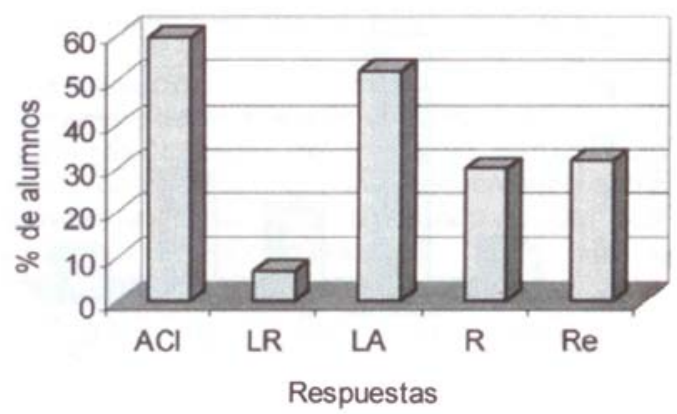

Fig. 6: Opiniones de los alumnos sobre las etapas necesarias para el estudio

\section{La Química Descriptiva como Objeto de Aprendizaje}

El proceso de enseñanza- aprendizaje de la Química descriptiva en general, y de la Química Inorgánica, presenta dificultades, debido a diversas causas, algunas de las cuales están relacionadas con las características de la disciplina.

Entre las causas que guardan relación con el tipo de disciplina, son destacables las siguientes: el tipo de contenidos fácticos a aprender; la creciente amplitud de los contenidos y la necesidad de seleccionar y sistematizar los mismos para no incurrir en el enciclopedismo. Debido a estas características, es de fundamental importancia que el alumno de Química descriptiva esté capacitado para aprender el tipo de contenidos propio de esta disciplina, y para ello, que sea capaz de estudiar. 


\section{Concepciones del Alumno de Química Descriptiva}

La información obtenida a través distintos instrumentos permite inferir que el estudiante de Química:

- No tiene conciencia de sus propios procesos y estrategias mentales.

- Como consecuencia, tiende a veces a engañarse a si mismo respecto a su forma de estudiar.

- Se considera responsable de su éxito o fracaso en el aprendizaje, ya que menciona como la causa más importante de su fracaso la falta de estudio, y como factores más importantes para el éxito, el "saber estudiar"y la dedicación.

- Pese a conocer la importancia de "saber estudiar" no se ha planteado la necesidad de adquirir dicho saber.

- En general no posee una adecuada metodología de trabajo intelectual, como lo evidencian los resultados del test de Pózar.

- Manifiesta aplicar técnicas de elaboración de los conocimientos; pero al ser interrogado acerca de las mismas, no son capaces de describirlas.

- Tiende a asociar el conocimiento de la disciplina con el requerimiento de una excelente memoria.

- Desconoce la importancia de las técnicas de asimilación, como surge de los resultados del test de Pózar, y del bajo porcentaje de alumnos que mencionan el "repaso" como una etapa relevante del estudio.

- Atribuye excesiva importancia al rol del profesor, y a la clase magistral, a la que considera la etapa más importante para su aprendizaje.

\section{El Estudio Eficaz}

Estudiar eficazmente implica lograr almacenamiento, elaboración y recuperación de la información. Para alcanzar estos objetivos pueden aplicarse distintas estrategias. Las estrategias de aprendizaje podrían ser definidas (Kirby, 1984; Nisbet y Schucksmith, 1987; Nickerson et al., 1987) como comportamientos planificados para seleccionar y organizar mecanismos motrices, cognitivos y afectivos con el fin de enfrentarse con éxito a situaciones de aprendizaje. Sin embargo, no basta con conocer estrategias para lograr un aprendizaje fructífero. En efecto, se ha comprobado que son más productivos los aprendizajes precedidos por una reflexión crítica de los estudiantes sobre sus propias prácticas, que aquellos en los que se siguen prescripciones de "buenas" prácticas de aprendizaje (Main, 1985). Por lo tanto, al uso deliberado de estrategias adecuadas de estudio, debe agregarse un nuevo componente, la metacognición, que conduce a tomar conciencia de los propios procesos intelectivos. La metacognición permite que los estudiantes controlen de forma consciente la aplicación de las técnicas de estudio. Para el alumno de Química descriptiva, es siempre provechoso analizar sus métodos de estudio, ya que los mismos pueden no ser adecuados para esta disciplina, aunque hayan sido eficaces para encarar el estudio de otras ramas de la ciencia.

\section{Aplicación del Test y Metacognición}

La información que se brindó a los alumnos respecto a los resultados del test de Pózar fue para muchos de ellos el punto de partida para iniciar el proceso de metacognición. La discusión grupal de dichos resultados en un taller permitió sugerir a los estudiantes diferentes técnicas para sistematizar su trabajo intelectual, asesorarlos sobre dichas técnicas y conducirlos a la práctica de las mismas.

\section{CONCLUSIONES}

Los resultados obtenidos permitirían afirmar que el estudiante medio de Química Inorgánica: no aplica en su estudio de Química descriptiva metodologías adecuadas de trabajo intelectual, no reflexiona sobre sus hábitos y estrategias de estudio y considera que es importante saber estudiar. 
La situación descripta puede atribuirse en parte a las prácticas usuales de enseñanza-aprendizaje, que no contribuyen a que el alumno tome conciencia de sus limitaciones para el trabajo intelectual.

Tama (1986) identifica tres áreas de atención educativa:

- Enseñar a pensar, implantar y desarrollar en los estudiantes un conjunto de habilidades cognitivas.

- Enseñar sobre el pensar, para que el estudiante tome conciencia de sus procesos estrategias mentales (metacognición).

- Enseñar sobre la base del pensar, incorporando objetivos de aprendizaje relativos a las habilidades cognitivas.

En general, los docentes brindan a sus alumnos esmerada atención sólo en la primera de las áreas mencionadas. Son escasos, en cambio, los que consideran importante conducir a los estudiantes a la metacognición. En cuanto a los objetivos de aprendizaje referentes a las habilidades cognitivas, no son formulados (al menos en forma explícita). Por otra parte, pruebas, exámenes y otros instrumentos usuales no están diseñados para evaluar procesos cognitivos o habilidades para el estudio.

El análisis de los resultados obtenidos evidencia la necesidad de metodologías educativas que favorezcan la adquisición de hábitos de estudio eficaces y contribuyan a que el alumno:

- Se sienta responsable se su propio aprendizaje, e internalice la necesidad de adquirir metodologías adecuadas para el estudio de Química descriptiva.

- Realice el proceso de metacognición.

- Conozca y practique metodologías de trabajo intelectual.

- Adquiera hábitos de estudio que le permitan mejorar su rendimiento.

Entre las estrategias aplicables para lograr estos objetivos, pueden proponerse las siguientes:

- Introducir actividades que promuevan la reflexión del alumno sobre sus mecanismos de aprendizaje.

- Posibilitar la discusión de los procesos y tácticas de aprendizaje de otros.

- Ofrecer al estudiante la oportunidad de conocer y practicar estrategias t técnicas metacognitivas relacionadas con la adquisición, ampliación y aplicación de la información.

- Apoyar la aplicación selectiva de las estrategias de aprendizaje que cada individuo haya identificado como eficaces.

\section{REFERENCIAS}

Butler, L. y J. Harrod; Química Inorgánica -Principios y Apllicaciones- Addison Wesley Iberoamericana-USA (1992).

Kirby, J; Cognitive Strategies and Educational Performance, Academic Press, Nueva York (1984).

Nisbet J. y J. Schucksmith; Estrategias de Aprendizaje, Santillana, Madrid (1987).

Nikerson, R., D. Perkins y E. Smith; The Teaching of the Thinking, Lawrence Eribaum Assoc., New Jersey (1987)

Main, A.; Reflection and the Development of Learning Skills. En: Boud, D.; Keogh, R.; y Walker, D.; Reflection: Turning Experience into Learning. Londres, Kogan Page (1985).

Tama, M.: Thinking Skills: A Return to the Content Area Classroom - Comunicación presentada en el "Meeting of the International Reading Association" Filadelfia (Abril 1986). 